\title{
Innovación en la comunicación científica y académica: las revistas electrónicas
}

\author{
Alfonso López Yepes ( ${ }^{*}$ ) \\ Catedrático de Documentación \\ Universidad Complutense de Madrid \\ E-mail: alopez@eucmax.sim.ucm.es
}

\subsection{Resumen}

Las nuevas tecnologías de la información y sus aplicaciones en la era digital están provocando importantes cambios en todos los ámbitos profesionales y en todas las formas de organización cultural y social. En este contexto se produce una innovación en la comunicación científica y académica, favorecida por la proliferación de revistas eletrónicas en soporte cederrón y accesibles a través de Internet o diseñadas para ser consultadas exclusivamente en Internet. Se comentan las ventajas que conlleva la edición digital de revistas científico-universitarias frente a las publicaciones tradicionales, así como la utilización de las redes de comunicaciones para su consulta y difusión. En el ámbito documental se presenta el número especial de la revista Cuadernos de Documentación Multimedia, dedicado de forma monográfica a estudiar las últimas tendencias en soportes multimedia y documentación informativa. Se publica en tres soportes -impreso, cederrón y red, y cuenta con la colaboración de investigadores de dieciséis universidades.

Palabras clave: Documentación digital. Documentación informativa. Documentación multimedia. Edición digital. Revistas electrónicas. Sociedad de la información.

\subsection{Abstract}

The new technologies of information and their applications in the digital age are provoking important changes in all the professional environments and in all the forms of cultural and social organization. In this context an innovation in

(*) Director del Servicio de Documentación Multimedia y de la revista "Cuadernos de Documentación Multimedia". Departamento de Biblioteconomía y Documentación. Facultad de Ciencias de la Información de Madrid. (http://www.ucm.es/info/multidoc).

Scire. 5 : 2 (jul.-dic. 1999) 11-21. 
scientific and academic communication appears, favored by the proliferation of electronic journals in CD-ROM and Internet. The advantages this approach carries in comparison with the traditional publications is examined, and also the particulars of the usage of communication networks for their access and dissemination. In the field of Documentation, the case of the special issue of Cuadernos de Documentación Multimedia is presented. This issue is monographicaly devoted to the last advances in multimedia and mass media documentation. It is published on three media — printed, CD-ROM and Internet- and compiles the contributions of researchers from sixteen universities.

Keywords: Digital documentation. Mass media documentation. Multimedia Documentation. Digital publishing. Electronic journals. Information Society.

\section{Sociedad documental, sociedad multimedia, sociedad-red}

Puede afirmarse, recién entrados en 1999 que las nuevas tecnologías de la información han provocado definitivamente un cambio profundo en los modos de trabajo de cualquier tipo de profesional y en cualquier labor que se realiza en la denominada sociedad de la información. Es un hecho, unánimamente aceptado, que se ha producido una transformación social y cultural que está originando un nuevo ámbito industrial y unas nuevas formas culturales, de relación y de intercomunicación personal y grupal. La mencionada transformación continúa afectando a todos los ámbitos profesionales y sus campos de aplicación: comercio, finanzas, servicios, entretenimiento, formación, medios de comunicación social, edición-publicación.. y, obviamente, el ámbito de los archivos, bibliotecas y centros y servicios de información y documentación. Todavía se precisa una fase de adaptación a dichos cambios originados por los últimos desarrollos de la tecnología digital, hasta la culminación, en todos los niveles de actuación, del proceso de integración ya iniciado.

La actual "sociedad de la información" (también denominada "sociedad multimedia" y más recientemente "sociedad documental" e incluso "sociedadred"), considerada como nuevo medio de comunicación, plantea una serie de cuestiones actuales sobre la influencia de las nuevas tecnologías de la información en el entorno del individuo y supone una reflexión sobre la transformación de nuestra vida cotidiana y de las formas de organización social. El debate sobre el futuro inmediato que propugnan las nuevas tecnologías multimedia en la era digital permanece abierto.

Por otra parte, el desarrollo imparable e incuestionable de la microinformática, de las memorias ópticas de almacenamiento y de las redes de comunicación, deberá complementarse permanentemente con nuevos sistemas, más inteligentes, de almacenamiento holográfico, de compresión y de producción de informa- 
ciones, de mejor calidad en la visualización, lectura y audición, de mayor velocidad en el acceso a los datos. Porque actualmente la demanda de información es tan inmensa, la generación de documentación tan ilimitada, que el sistema tradicional, basado en soportes impresos, se muestra físicamente incapaz de controlar tal cúmulo de datos.

Asimismo, la tendencia progresiva a la consulta, producción, almacenamiento, recuperación y difusión eminentemente digital de información, y al uso de las redes que posibilitan el acceso y gestión virtual de la documentación de cualquier ámbito profesional está provocando la aparición de una "sociedad documental". En ella se generan, comunican y localizan documentos en entornos digitales, que posibilitan en muchos casos el acceso a informaciones con contenidos multimedia (texto, imagen fija, audio y vídeo). De ahí que también se utilice el término de "sociedad multimedia", como consecuencia de esa proliferación de documentos multiformes.

En fin, la evolución de las redes, más concretamente de Internet, ha sido tan grande que los datos estadísticos que se relacionan a continuación prueban el fenómeno que ha supuesto la introducción de la red de redes en el entorno profesional e incluso familiar. La radiodifusión tardó cerca de 30 años en llegar a casi 60 millones de hogares en Estados Unidos; la televisión logró idéntica penetración en 15 años, y la televisión por cable lo hizo en 10 años. Internet lo ha conseguido solamente en tres, según datos del Congreso sobre Internet celebrado en España en 1998.

No obstante, como resulta obvio que más datos no suponen necesariamente mejor información, sino más "ruido" informativo, y que a mayor cantidad de información, mayor necesidad de búsqueda y sistematización documental... se precisa disponer, en consecuencia, de motores cada vez "más inteligentes" de tratamiento documental y de localización de informaciones contrastadas, y de profesionales cada vez más expertos en la localización, producción, recuperación y difusión de informaciones.

Por consiguiente, los comunicadores, los profesionales de la información en general, los documentalistas tienen un nuevo cometido que cumplir en la era de la información electrónica, caracterizada por la digitalización, la integración, la globalización, como una inmensa red universal de datos o Red Mundial. La red de redes, Internet (y sus derivaciones, Intranet/Extranet), se presenta en consecuencia como un nuevo medio de comunicación, como el actual instrumento por excelencia para la recepción, consulta, producción y difusión de informaciones de todo tipo.

Hasta tal punto que se tiende progresivamente al establecimiento en todos los ámbitos profesionales de verdaderos "sistemas interactivos en línea" para el 
acceso a la información que circula a través de las infopistas, una vez haya sido resuelto a nivel global el problema del ancho de banda adecuado. Nos encontramos en los albores de la era digital, en un momento en que las tecnologías disponibles de digitalización de imagen y sonido, robótica y manejo de grandes bases de datos y redes permiten a cualquier profesional en cualquier ámbito de trabajo, consultar todo tipo de informaciones, producir información propia, digitalizar su material e integrarlo en un complejo sistema de acceso y difusión remotas.

Actualmente, en todos los foros especializados se habla de digitalización y de redes digitales, hasta el punto de que también en boca de comunicólogos y sociólogos se encuentra el tema: José B. Terceiro manifestaba muy recientemente, al referirse al término "digitalismo", que éste representa "la cultura nacida del entorno digital, que supondrá una forma original de organizar la vida como consecuencia de las aplicaciones de los modernos instrumentos de la información" (1). Por su parte, Manuel Castells (1999) señalaba, al referirse a las tecnologías de red, que presentan hoy en día una relación muy estrecha con los conceptos de empresa-red y sociedad-red, que "las tendencias sociales y empresariales apuntan hacia la estructura en la red como estructura dinámica, competitiva, eficaz, flexible y operativa; es decir, se basan en el desarrollo de una lógica de redes".

Todavía más recientemente (18 febrero1999), abundando en este proceso incontenible de la digitalización a nivel global, leemos que la gran librería virtual española "bol.com" (de Books On Line, el equivalente hispano de Amazon) pondrá un millón de títulos en castellano en la red a partir del próximo mes de septiembre (Moret, 1999).

\section{Edición digital versus edicion tradicional: Internet y las revistas digitales científico-universitarias.}

La proliferación de publicaciones electrónicas ha supuesto un cúmulo de posibilidades en la producción y difusión de informaciones. El ámbito de la investigación universitaria se ha visto considerablemente enriquecido merced a la edición digital de los trabajos y su conocimiento a través de las redes de comunicación (Aguirre, 1997-1998).

Diversos aspectos deben ser contemplados en relación con la producción digital que, a diferencia de los métodos tradicionales de publicación utilizados hasta ahora, presenta unas características económicas, fundamentalmente. La edición digital supone un abaratamiento muy importante de la edición, puesto que posibilita la difusión de un mayor volumen del trabajo investigador, y como consecuencia el aumento de productividad de los investigadores en su conjunto, paliando así los problemas derivados de la falta de espacio físico y, en consecuencia, el abaratamiento de los gastos de edición.

Scire. 5 : 2 (jul.-dic. 1999) 11-21. 
Por otra parte, la producción editorial universitaria adolece de una lentitud considerable, caracterizada por tres notas: se edita artesanalmente (los departamentos se ocupan de sus propias publicaciones); la producción universitaria en su conjunto se agrupa bajo un mismo sello editorial, y, en tercer lugar, se establecen acuerdos con empresas editoriales privadas, lo que supone algún tipo de dependencia. La producción investigadora en el ámbito privado conlleva servidumbres materializadas, en unos casos, en la necesidad de ajustar la producción a un mercado más amplio (se favorece la edición de un tipo de publicaciones concretas), y en otros, en la reducción de notas, aparatos críticos, etc.: condicionantes que todo investigador ha padecido en algún momento.

Otro aspecto económico a tener en cuenta es que la edición universitaria o científica es cara, debido fundamentalmente a la reducción en su tirada y al deseo por parte de sus editores de que las revistas sean adquiridas por las instituciones universitarias o con fondos de investigación. De otro lado, el precio tan elevado de la suscripción a revistas supone una carga considerable para las bibliotecas (especialmente), sometidas siempre a presupuestos reducidos que provocan la inevitable restricción en el establecimiento de dichas suscripciones. La consecuencia es que numerosas publicaciones periódicas universitarias se sienten "heridas" al ser pocas las instituciones suscritas a las mismas.

Para paliar de algún modo esta problemática se practica el intercambio, lo que conlleva que parte del presupuesto se libera, no comprando revistas sino cambiándolas por las que editan otras universidades: de esta forma se asegura la difusión de muchas revistas a las que no podría darse salida, contribuyendo a su conocimiento y utilización.

De otro lado, afortunadamente, las redes de comunicación y el buen uso de sus muchas posibilidades, puede favorecer considerablemente la producción y difusión del conocimiento investigador que se realiza en los ámbitos universitarios. Conviene recordar aquí que las infopistas constituyen un triple soporte informativo: son un medio de edición, de difusión y de comunicación, y que estas tres funciones facilitan considerablemente la producción científica. Me permito reproducir a continuación, a pesar de su larga extensión pero de gran interés, lo que ha escrito Joaquín Aguirre (2) (Ibidem, p. 165-167) al respecto:

“...Como "medio de edición" permite: 1) que los investigadores tengan un mayor control sobre los procesos editoriales, ya que pueden ser ellos mismos los que realicen la mayor parte de las tareas; 2) evitar las limitaciones de espacio que suelen imponer las editoriales. "Espacio" es papel y "papel” es dinero. La edición digital no necesita, como es obvio, papel alguno y desaparecen los costes derivados de este importantísimo capítulo y de los manipulados consiguientes; 3) que el material sea modificable o ampliable. En la publicación científica es muy importante la posibilidad de establecer distintas fases en los documentos. Determinados documentos se

Scire. $5: 2$ (jul.-dic. 1999) 11-21. 
encuentran en proceso de revisión y se distribuyen para obtener opiniones o contrastes antes de su redacción definitiva. La red es un medio perfecto para este tipo de sistemas.

Como "medio de difusión" permite: 1) solventar el problema de las bajas tiradas. La edición electrónica en red no entiende de "tiradas"; simplemente no existe el concepto, ligado al mundo del papel; 2) saltar las barreras de la distribución deficiente del material científico. La edición digital en línea no requiere distribuidoras; la red es su medio natural y está disponible para todos sin necesidad de intermediarios; 3) que no existan "números atrasados" ni devoluciones. La revista puede mantener todo su material en línea a disposición de sus lectores; 4) la distribución no requiere ningún proceso por parte de los editores. Son los lectores los que acceden a la información y no la información la que ha de llegar a los lectores. Es decir, el concepto de distribución desaparece tal como se concibe en el mundo impreso; 5) la red incorpora sus propios mecanismos de publicidad: la información en la red es localizable por el simple hecho de estar ahí, y se pueden realizar acciones para dar a conocer las publicaciones en línea. La principal tarea de la distribución en este medio se puede resumir en la localización de los lugares comunes y en la notificación a los mismos de la publicación. Si tiene interés para ellos, la incluirán en su selección de recursos específicos. Aunque no la recogiera ninguna otra página de la red, siempre quedaría a los posibles interesados el recurso de las búsquedas temáticas o por palabras clave que le llevarían directamente al artículo de sus pesquisas. Sería importante que se generalizara el hecho de que las universidades dispusieran de lugares específicos en la red para agrupar las fuentes, organizando sus recursos para, en segunda instancia, establecer esas concentraciones por áreas que facilitaran los accesos.

Como "medio de comunicación" permite: 1) el contacto directo e inmediato con los lectores. La inclusión de las direcciones de correo electrónico de los autores permite el debate, la matización, la crítica, el comentario, etc. Muchas comunidades científicas, especialmente en campos como la física, la astronomía, la medicina y similares, se mantienen en contacto gracias a las redes de comunicación a través de foros de discusión, boletines o el simple correo electrónico; 2) una mayor facilidad para la localización de especialistas en campos afines. La red permite localizar fácilmente a personas que trabajan en nuestros mismos campos. Esto es enriquecedor para nuestro trabajo pues permite el intercambio de ideas y datos...".

Aunque también conviene destacar que la red entraña inconvenientes, derivados básicamente del propio medio en sí, y que afectan fundamentalmente a aspectos como la conservación de los materiales que circulan por la misma, y los cambios (la evolución permanente) que la caracterizan.

Asimismo, la comunidad académico-científica en su conjunto tendrá que adaptarse a los cambios que está provocando la recién estrenada era digital, y no sólo basarse en los márgenes que establece la edición impresa. Hasta que no se llegue a este punto, las revistas en línea estarán, sin duda, en inferioridad de condiciones.

Scire. 5 : 2 (jul.-dic. 1999) 11-21. 


\section{Las revistas electrónicas de documentación y Cuadernos de documentación multimedia: una innovación en la comunicación científica y académica.}

Las publicaciones electrónicas constituyen, por consiguiente, una gran fuente de información y difusión del saber, contribuyendo a la rápida divulgación de conocimientos e ideas. Si las publicaciones tradicionales fueron concebidas para transmitir avances científicos, los sistemas telemáticos mantienen esa función, al tiempo que suponen una nueva concepción en cuanto a distribución y nuevos modos de consulta y difusión de la comunicación científica y académica, como ha sido puesto de manifiesto en el epígrafe anterior.

Precisamente se hace eco de ello la muy reciente publicación de un trabajo de Angela Sorli y José Antonio Merlo (1998) en torno a las publicaciones electrónicas españolas sobre Archivística, Biblioteconomía y Documentación. Según estos autores las revistas presentes en la red, algunas de ellas publicadas exclusivamente para ser leídas a través de Internet, contribuyen poderosamente a la divulgación de la ciencia puesto que su acceso y difusión se realiza a nivel global. Su presencia ya es tan destacada que hasta podrían clasificarse en cinco apartados: revistas y boletines de asociaciones profesionales; publicaciones elaboradas por bibliotecas o centros de documentación; ediciones periódicas editadas por universidades o centros de formación; revistas de instituciones de ámbitos documentales; y boletines de empresas del mundo de la información.

Conviene señalar que unas ofrecen texto completo de las colaboraciones que recogen, y en otros casos solamente relación de autores, títulos de artículos (a veces también materias) y referencia del número en que fue publicado el trabajo y las páginas en las que aparece, así como resúmenes (a veces en varios idiomas) y otras informaciones, intercambio de experiencias entre centros afines, etc.

En el ámbito de la Documentación informativa o de medios de comunicación social, acaba de publicarse (se distribuye desde enero de 1999) un número especial (6-7, 1997-1998) de la revista Cuadernos de Documentación Multimedia, publicación periódica que dirige desde 1992 el autor de estas páginas. La revista, que se edita en el ámbito del Servicio de Documentación Multimedia de la Universidad Complutense de Madrid, dependiente desde 1993 del Departamento de Biblioteconomía y Documentación de la Facultad de Ciencias de la Información (3), se encuentra presente en Internet desde su número 3 (1994).

El trabajo que se lleva a cabo en el Servicio de Documentación Multimedia - cuyas últimas realizaciones son reseñadas precisamente en este especial de Cuadernos (López Yepes, 1997-1998) — contribuye sin duda a esa innovación en la comunicación científico-académica que se ha originado recientemente, apoyada en el uso de las últimas tecnologías y que se encuentra en estrecha conso-

Scire. $5: 2$ (jul.-dic. 1999) 11-21. 
nancia con la filosofía del Servicio. Conviene recordar que sus objetivos son, desde su puesta en marcha: 1. Proporcionar a investigadores y estudiosos información sobre comunicación social y sus especialidades mediante instrumentos automatizados de recuperación de información; 2. Difundir las últimas tendencias que presenta la tecnología digital y sus aplicaciones en el ámbito de la comunicación de masas, y en la empresa informativa en general; 3. Informar acerca de proyectos y elaboración de bases de datos nacionales e internacionales, con contenidos multimedia especialmente, sobre temáticas comunicacionales a cargo de instituciones especializadas; 4 . Recopilar, analizar, recuperar y difundir bases de datos multimedia en materia. de comunicación audiovisual, mediante instrumentos apropiados de hardware y software de gestión documental; 5. Producir bases de datos multimedia propias o en colaboración con otras empresas informativas públicas y privadas; y 6 . Contribuir a la formación e investigación en documentación informativa mediante el uso de metodologías innovadoras sustentadas en la tecnología digital.

Cuadernos de Documentación Multimedia presenta en esta ocasión un monográfico, de carácter extraordinario, sobre "Multimedia y Documentación informativa: Tendencias actuales", que reúne, en cuatro bloques temáticos, las aplicaciones más recientes de las nuevas tecnologías en los ámbitos documentales de medios de comunicación, materializadas en 37 colaboraciones. Los contenidos tratados abordan las siguientes temáticas: nuevas metodologías y producción de materiales docentes en la enseñanza; formación e investigación en documentación informativa: hacia una enseñanza con contenidos multimedia; edición-producción multimedia; y documentación multimedia en medios de comunicación social. Se completa la revista con un Anexo que recoge 42 estudios documentales multimedia sobre medios informativos españoles, elaborados por Licenciados en Comunicación Audiovisual y Licenciados en Documentación por la Universidad Complutense.

Han colaborado en esta última entrega prestigiosos profesionales españoles, entre profesores e investigadores universitarios (16 universidades) y responsables de documentación de medios de comunicación impresos, audiovisuales y electrónicos (10 empresas informativas). Se trata, en consecuencia, de un trabajo de integración de especialistas, de convergencia de temáticas documentales, como corresponde a esta nueva era de la información.

Finalmente, debe destacarse que la publicación — sujeta a suscripción (4)— ha sido editada en tres soportes, en papel, en cd-rom y accesible a través de Internet, pudiendo afirmarse que es la primera revista española (en soporte convencional y electrónico) sobre documentación que presenta esta triple posibilidad de lectura y consulta. Incluye información multimedia que se distribuye de las siguiente manera: 450 páginas de texto, 100 ilustraciones fijas y 55 minutos 
de vídeo digital (de información audiovisual digitalizada sobre formación en documentación y sobre documentación en la empresa informativa). Sobre el primer aspecto se incluye un vídeo de 10 minutos (Los estudios de Documentación en la Universidad Complutense de Madrid), escrito, realizado y postproducido en una plataforma AVID de edición no lineal, dedicándose el resto del minutado a la información audiovisual obtenida en las instalaciones de diversos medios informativos. La consulta de los contenidos puede gestionarse en estos momentos mediante un motor de búsqueda documental, producido por la empresa OCS, que permite un alto grado de relevancia en la recuperación de informaciones (5).

En suma, nos situamos en estos momentos en plena innovación científica y académica, pudiendo afirmarse que están dándose los primeros pasos en cuanto a la enseñanza no presencial de la documentación a través de la red, apoyándose en soportes electrónicos complementarios —nos referimos por ejemplo al "I Curso de Postgrado sobre Documentación Digital" (6)—, con la propuesta asimismo de "Servicios Web" tanto para la gestión documental de recursos, como para la formación en biblioteconomía y documentación.

Se empieza a trabajar, como decimos, en la configuración de los denominados "Servicios Web de información y documentación", basados en tecnología World Wide Web de Internet, por tanto dotados de una configuración estándar, abierta y generalizada a nivel mundial, y que posibilita el acceso y difusión de información multimedia (texto, imagen fija, audio y vídeo). Funcionan en entornos Intranet/Internet en el ámbito de la institución (biblioteca, centro de documentación, etc.), lo que supone facilidad de consulta, compartición y protección de la información propia y acceso al mismo tiempo a todos los datos -información ajena- que circulan a través de las redes. En este sentido, el autor de estas páginas ha presentado recientemente (17 febrero 1999) en Salamanca, una comunicación sobre documentación audiovisual y cinematográfica, en el "III Congreso Internacional de Cultura y Medios de Comunicación". Asimismo, ha participado en un Teleseminario virtual sobre Documentación audiovisual organizado durante la última semana del mismo mes de febrero por el CETE (Centro de Entrenamiento de Televisión Educativa, de la UNAM- Universidad Nacional Autónoma de México), transmitido a través del canal temático Edusat (12).

La utilización de Servicios Web en la formación en biblioteconomía y documentación es una propuesta planteada por García Marco (1997-1998, precisamente en el número especial de Cuadernos de Documentación Multimedia al que se acaba de hacer referencia), puesto que se trata de:

“... Un tipo de servicios que constituye un medio integrado de publicación, archivo, organización, recuperación y divulgación científica y como tal servicio de información, no cumple tan sólo funciones de publicación externas, sino también internas (Intranets), así como instrumentos de comunicación privilegiada con los usuarios y

Scire. $5: 2$ (jul.-dic. 1999) 11-21. 
proveedores estables de la organización (Extranets). Por todo ello, cabe augurar que la World Wide Web se convertirá en la plataforma ideal para soportar las memorias documentales de las instituciones. Gracias a estas característica, un servicio de este tipo se transforma en la memoria viva de la organización, tanto de cara a su funcionamiento interno como a su comunicabilidad social. En su ámbito, el documentalista puede aportar conocimiento científico y experiencia en su desarrollo y más concretamente en los siguientes apartados: 1 . Estudios de demanda y necesidades de información científica; 2. Estudios de hábitos de comunicación y acceso de los usuarios; 3. Investigación y diseño de políticas de información científica y de las relaciones con otros sistemas de información; 4. Planificación de los contenidos del servidor, de su estructura interna y de sus medios de acceso y difusión; 5. Tratamiento del documento científico electrónico (meta-información): catalogación, indización y resumen; 6. Diseño y mantenimiento de lenguajes documentales universales y, sobre todo, especializados con los que sistematizar y proporcionar acceso eficaz y "sin ruido" al servicio; 7. Diseño y mantenimiento de bases de datos de referencias a documentos científicos electrónicos.; 8. Diseño y mantenimiento de algoritmos de optimización de las búsquedas; 9. Gestión de la biblioteca de recursos (textos en distintos idiomas, imágenes, vídeos, etc.); 10. Gestión documental del servidor: versiones, selección, expurgo, archivo definitivo, y 11. Gestión de los requisitos legales de la publicación científica"(7).

Por último, debe destacarse que también está contribuyendo en la innovación científica y académica española la labor desarrollada por la Red Iris con el mantenimiento de listas de discusión en torno a numerosos ámbitos del conocimiento científico y académico español, y especialmente en relación con los archivos, bibliotecas y centros de documentación: IWETEL, MUSICDOC, CINEDOC, etc.(8).

\section{Notas}

(1) José B. Terceiro (1999) bautiza como "digitalismo" los grandes cambios provocados por las nuevas tecnologías.

(2) Joaquín María Aguirre Romero es profesor del Departamento de Filología Española III y Vicedecano de Desarrollo Tecnológico y Prácticas en la Facultad de Ciencias de la Información de la Universidad Complutense de Madrid, y director de la revista electrónica Espéculo (http://www.ucm.es/OTROS/especulo/).

(3) Sobre el origen y evolución del Servicio de Documentación Multimedia puede consultarse mi libro más reciente (López Yepes, 1997). El Servicio está accesible a través de http://www.ucm.es/info/multidoc/multidoc, y en el Dpto. de Biblioteconomía y Documentación (en http://www.ucm.es/info/multidoc).

(4) La suscripción al soporte impreso es de 3.500 ptas., y al soporte impreso más el CDROM, de 5.000 ptas. Su punto de venta en España está localizado en Librería Fragua, calle Andrés Mellado, 64. 28015 Madrid. Tel. 915491806 y 91 5442297. Fax: 91 5491806. E-mail: fragua@fragua.com.

(5) URL: <http://www.ocs.es/rware/ocs_demo.html>.

Scire. 5 : 2 (jul.-dic. 1999) 11-21. 
(6) Para mayor información sobre el "I Curso de Postgrado sobre Documentación digital", organizado entre febrero y mayo de 1999 por la Universidad Pompeu Fabra de Barcelona, y el cd-rom de apoyo docente denominado Biblioteca Digital (Véase URL: <http://camelot.upf.es/digital/>).

(7) La comunicación trata el tema "CineDocNet": Información y Documentación cinematográfica en red. Hacia un modelo de Servicio de Documentación audiovisual en línea". Disponible en: <http://www.comunicacion.teleline.es/comunicacion/ponencias/mesa5/yepes.htm>. Sobre el trabajo presentado en el Teleseminario mexicano puede verse: 〈http://www.sep.gob.mx/cete/index.htm>. (Ambas colaboraciones se encuentran en prensa en estos momentos).

(8) Véase: <http://www.rediris.es/list/info/iwetel.html, <http://www.rediris.es/list/ info/musicdoc.html, <http://www.rediris.es/list/info/cinedoc.html>. Sobre éstas y otras listas de distribución mantenidas por Red Iris véase además: <http://www.rediris.es/list/tema/tematic.es.html. Y sobre éste y otros aspectos de la documentación digital, véase López Yepes (1999).

\section{Referencias}

Aguirre Romero, Joaquín María. Las revistas digitales y la vida académica. // Cuadernos de Documentación Multimedia. 6-7 (1997-1998) 159-169.

Castells, Manuel (1999). La hora de Jini. // Ciberpaís. (11.2.1999) 9.

Gacía Marco, Francisco Javier (1997-1998). Servicios de información en la World Wide Web: una nueva frontera para los docentes del área de biblioteconomía y documentación”. Cuadernos de Documentación Multimedia. 6-7 (1997-1998) 105-107. URL: <http://www.ucm.es/info/multidoc/multidoc/revista>.

López Yepes, Alfonso (1997). Documentación informativa: Sistemas, redes y aplicaciones. Madrid: Síntesis, 1997. 178 p.

López Yepes, Alfonso (1997-1998). Seis años de proyectos y realizaciones en el Servicio de Documentación Multimedia de la Universidad Complutense de Madrid (19931998). // Cuadernos de Documentación Multimedia, 6-7 (1997-1998) 141-148. URL: <http://www.ucm.es/info/multidoc/multidoc/revista>.

López Yepes, Alfonso (1999). La Documentación digital. BCN Digital Punt de Connexió 1999. Barcelona, Centre de Cultura Contemporània, 1999, 30 págs. (En prensa). URL: <http://www.cccb.org/bcndigital/>.

Moret, Xavier (1999). La librería virtual “bol.com” pondrá un millón de títulos castellanos en la red. // Ciberpaís. Comercio. (18.2.1999)15.

Sorli Rojo, Angela y Merlo Vega, José Antonio (1998). Publicaciones electrónicas españolas sobre Archivística, Biblioteconomía y Documentación”. // Revista Española de Documentación Científica. 21 : 4 (octubre-diciembre 1998) 451-462. URL: <http://www.cindoc.csic.es/webpublic/revista.htm>.

Terceiro, José B. (1999). [Sin título]. // El País. Sociedad. (11.2.1999) 30.

Scire. 5 : 2 (jul.-dic. 1999) 11-21. 